\title{
Wavelength Shifts in Solid-State Circular Dichroism Spectra: A Possible Explanation
}

\author{
ETTORE CASTIGLIONI, ${ }^{1,2 *}$ SERGIO ABBATE, ${ }^{2}$ GIOVANNA LONGHI, ${ }^{2}$ AND ROBERTO GANGEMI ${ }^{2}$ \\ ${ }^{1}$ JASCO Corporation, Hachioji-shi, Tokyo, Japan \\ ${ }^{2}$ Dipartimento di Scienze Biomediche e Biotecnologie, Università di Brescia, viale Europa 11, 25123 Brescia, Italy
}

\begin{abstract}
We have devised an artificial sampling approach generating "absorption flattening" (AF) on the UV-Vis spectrum of a solution of a chiral compound: Tris (ethylendiamine) cobalt(III) chloride $\left(\Lambda-\mathrm{Co}(\mathrm{en})_{3} \mathrm{Cl}_{3}\right)$. We have observed a concomitant red shift of the $\mathrm{CD}$ maxima. Related $\mathrm{CD}$ and absorption spectra have been calculated from spectra recorded on diluted homogeneous solutions, thus monitoring the effect of $\mathrm{AF}$ on both types of data. Experimental data are in good agreement with calculated spectra. Simulations with suitable bandshapes show that the red shift of the $\mathrm{CD}$ spectrum is due to $\mathrm{AF}$. On the basis of these results, we conclude that $\mathrm{AF}$ is an important cause of distortions in CD spectra for inhomogeneous samples. Plans to compensate or at least to take into account this effect are presented. Chirality 19:491-496, 2007. @ @ 2007 Wiley-Liss, Inc.
\end{abstract}

KEY WORDS: circular dichroism; solid-state spectroscopy; absorption flattening

\section{INTRODUCTION}

Absorption flattening (AF) is a well-known, but often neglected, phenomenon ${ }^{1}$ considered mainly for absorption spectroscopies of biomolecules. ${ }^{2}$ It takes place when absorbing particles are not uniformly distributed in the total volume of the cell, and it is present when dealing with suspensions, membranes, etc., but happens also with solids dispersed in $\mathrm{KBr}$ or other similar matrices. IR spectroscopists should be familiar with it, when evaluating quality of pellets or accepting spectral compromises with easy-sampling diffuse reflectance accessories. In short, $\mathrm{AF}$ can be considered, and actually acts, as stray-light. Usually, though stray light in spectrometers is radiation of different wavelength from the one selected by the monochromator; in this case, it is radiation at the proper wavelength which may pass freely through the nonhomogeneous sample. The quantitative effects of $\mathrm{AF}$ are not easily estimated. We want here to consider also the effect of $\mathrm{AF}$ on $\mathrm{CD}$ spectra, a problem already reported in the literature. ${ }^{3,4}$ We consider here a simplified model in which the sample is not homogeneous, and consequently, the radiation is free to pass through it without interaction with sample chromophores in a certain percentage. Let us define $f$ by the ratio of the number of photons which interact with the absorbing sample and the total number of incoming photons. We now estimate the effect on absorption/transmittance and CD spectra as follows:

\section{Absorption}

The transmittance $T(\lambda)$ spectrum of the sample can be considered as the sum of the absorbed and the free-topass components.

$$
T_{\operatorname{Exp}}(\lambda)=f T_{\text {Sam }}(\lambda)+(1-f) T_{\text {Sol }}(\lambda)
$$

where $T_{\text {Exp }}$ is the expected transmittance of the inhomogeneous sample, $T_{\mathrm{Sam}}$ is the transmittance given by photons (C) 2007 Wiley-Liss, Inc. interacting with sample chromophores, $T_{\mathrm{Sol}}$ is the transmittance of photons passing through the solvent only, and $f$ is the factor defined earlier.

\section{Circular Dichroism}

CD (in conventional instruments based on electro-optic modulators) can be expressed as the ratio between the $\operatorname{AC}(\lambda)$ and the $\operatorname{DC}(\lambda)$ components. Indeed, the $\mathrm{CD}$ signal is defined as

$$
\mathrm{CD}=\left(I_{\mathrm{L}}-I_{\mathrm{R}}\right) /\left(I_{\mathrm{L}}+I_{\mathrm{R}}\right) \approx \mathrm{AC} / \mathrm{DC}
$$

where $I_{\mathrm{L}}$ and $I_{\mathrm{R}}$ are the intensities for left and right circularly polarized light. In practical terms, commercial instruments measures the $\mathrm{AC}$ component by a lock-in amplifier, while the DC component is kept constant all over the spectral range by the dynode feedback. ${ }^{5}$

Here again, the overall $\mathrm{AC}$ signal can be assumed to be just the superposition of the AC signals, and so we will have

$$
\mathrm{CD}_{\operatorname{Exp}}(\lambda)=\left[f \mathrm{AC}_{\mathrm{Sam}}(\lambda)+(1-f) \mathrm{AC}_{\mathrm{Sol}}(\lambda)\right] / \mathrm{DC}_{\mathrm{Exp}}(\lambda)
$$

where $\mathrm{CD}_{\operatorname{Exp}}(\lambda)$ is the expected $\mathrm{CD}$ of the inhomogeneous sample, $\mathrm{AC}_{\mathrm{Sam}}(\lambda)$ and $\mathrm{AC}_{\text {Sol }}(\lambda)$ are the $\mathrm{AC}$ component of the $\mathrm{CD}$ signal due to interacting and noninteracting photons, and $\mathrm{DC}_{\operatorname{Exp}}(\lambda)$ is the $\mathrm{DC}$ component of the inhomogeneous sample.

${ }^{\star}$ Correspondence to: Ettore Castiglioni, c/o JASCO Europe srl, via Confalonieri 25, 23894 Cremella (Lc), Italy.

E-mail: ettore.castiglioni@jasco-europe.com

Received for publication 16 January 2007; Accepted 23 February 2007

DOI: $10.1002 /$ chir.20404

Published online 16 April 2007 in Wiley InterScience

(www.interscience.wiley.com). 

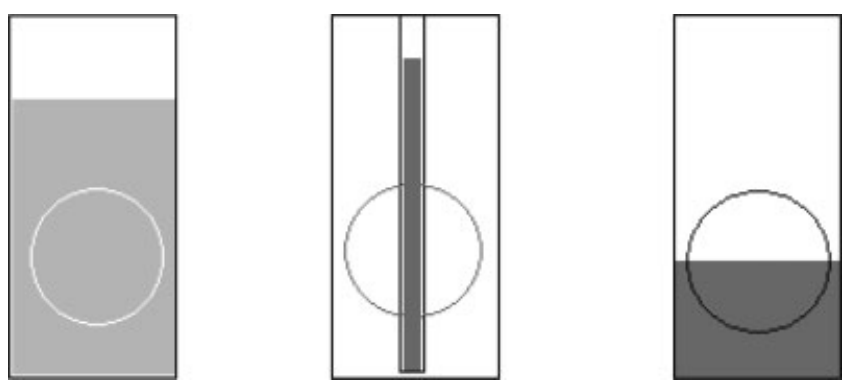

Fig. 1. Principle of the AF emulator: on the left light beam passing through the solution in a normal 10-mm wide cell; on the center, the sample is concentrated in the 2-mm aperture of the micro-cell, causing AF effects; on the right, variant of the AF emulator for short path cells. Part of the beam will pass above the sample.

As shown below, eq. 2 will allow us to take into account that the classical relationship $\mathrm{CD}=\mathrm{AC} / \mathrm{DC}$ is mainly distorted by the wrong high $T$ coming from the AF effect. We note that in general there is a good amount of published $\mathrm{CD}$ data exhibiting this phenomenon. As a work example we have chosen Tris(ethylendiamine)cobalt(III) chloride $\left(\Lambda\right.$-Co(en) ${ }_{3} \mathrm{Cl}_{3}$ complex) water solution. ${ }^{6}$ This compound has been previously used to compare spectra in solution and solid state, about which a few references are worth mentioning:

- An early JASCO Application Note ${ }^{7}$ : “... bromide rather than chloride complex in $\mathrm{KBr}$ pellet, main 490-nm band shifted in the red, compared to $\mathrm{H}_{2} \mathrm{O}$ solution spectrum. Reason not identified, but suspects on particle size, relative refractive indexes or absorption flattening ...";

- A paper from Kuroda ${ }^{8}$ reports the same red shift between $\mathrm{KBr}$ and aqueous solution spectra, even if the spectra are described as similar;

- In one paper ${ }^{9}$ by one of us, introducing the use of diffuse reflectance sphere for $\mathrm{CD}$, we observed the same
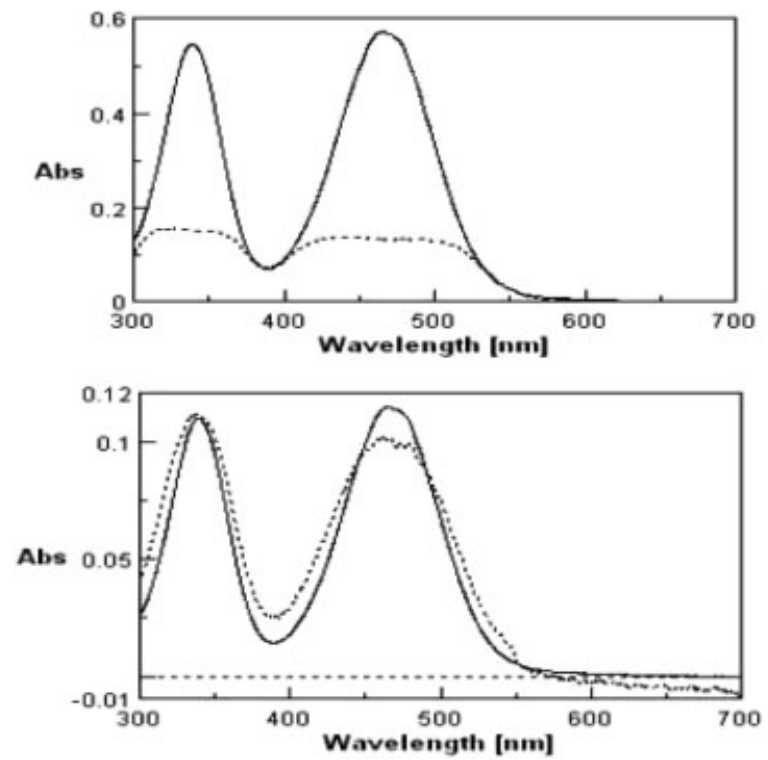

phenomenon with sample powder dispersed on a transmitting surface or pressed in CsI pellets, either in transmission mode or by diffuse reflectance. A relation between the red shift and sample concentration was observed for spectra obtained in pellet, but was not justified;

- In a further paper ${ }^{10}$ the same shift was noticed in diffuse reflectance mode, when diluting sample in $\mathrm{KBr}$ powder: the observed red shift increases with sample concentration going from $0.3 \%$ to $100 \%$. This was explained as a sort of "solvent or proximity effect."

\section{MATERIALS AND METHODS}

We emulate an AF sample in the following way: we prepare a conveniently concentrated $\left(\approx 3.65 \times 10^{-3} \mathrm{M}\right)$ aqueous solution of the $\Lambda-\mathrm{Co}(\mathrm{en})_{3} \mathrm{Cl}_{3}$ complex (kind gift of Prof. Sven E. Harnung of University of Copenhagen). Reference spectra have been first taken in standard 10-mm pathlength quartz cells, which are $10-\mathrm{mm}$ wide; the sam-

Fig. 2. Top: absorption (left) and CD spectrum (right) of aqueous solution of the $\Lambda$-Co(en) $)_{3} \mathrm{Cl}_{3}$ complex in standard cell (-) and with $5 \times$ concentration in the microcell (- - ). Bottom: absorption (left) and CD spectrum (right) of diluted sample in the microcell (- - -) compared to calculated normal cell spectra for $5 \times$ dilution $(-)$.

Chirality DOI 10.1002/chir 

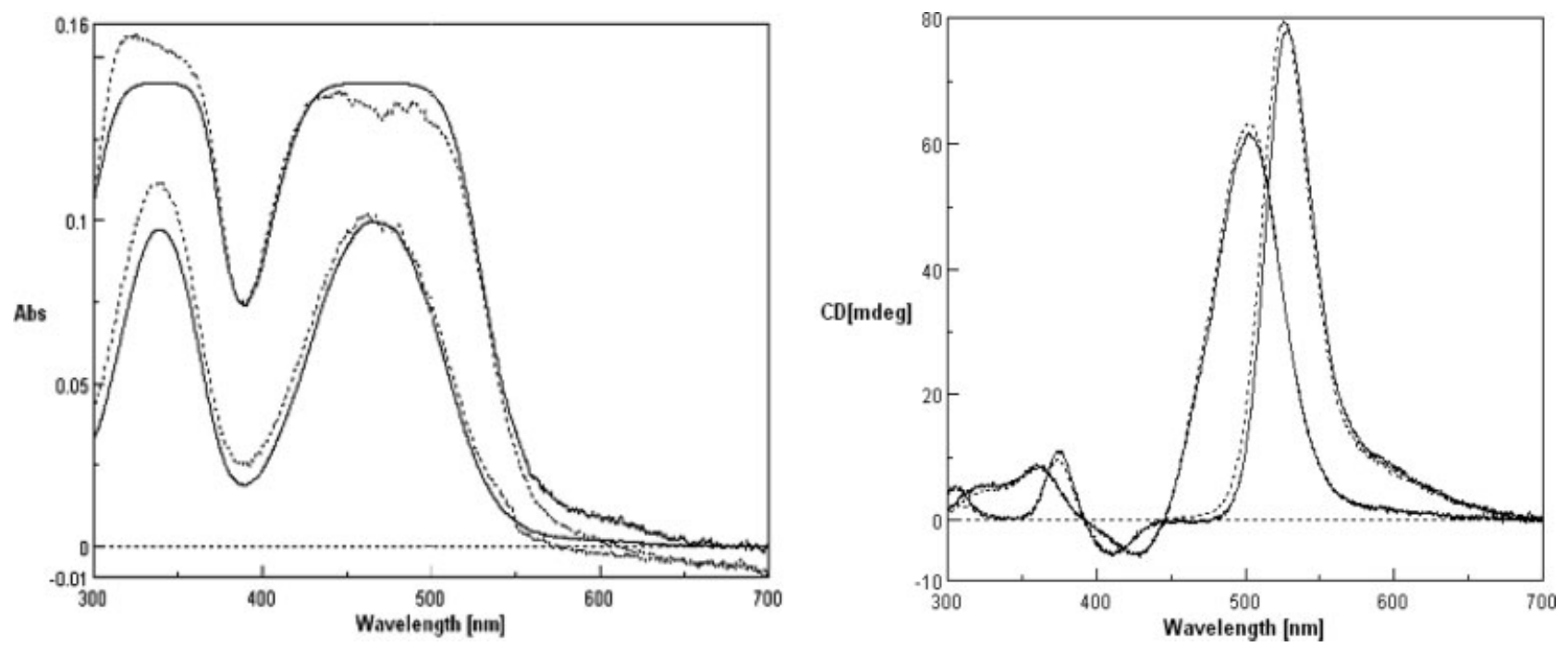

Fig. 3. Left: computed absorption spectra in microcell for the two concentrations of Figure $2(-)$ compared to experimental ones (- - -). Right: computed $\mathrm{CD}$ spectra in microcell for the two concentrations of Figure $2(-)$ compared to experimental ones (--).

ples for monitoring AF effects have been prepared by filling them in a 10 -mm pathlength rectangular quartz micro cell (inside width $2 \mathrm{~mm}$ ) with five times more concentrated solutions, to maintain the same quantity of chiral substance in the two cells. In this mode, the sample beam ( $\cong 9 \mathrm{~mm}$ diameter) is only partially passing through the solution, while most of it is free to pass through the transparent walls of the cell (Fig. 1, left and center); in this way, we emulate a sample which is not uniformly distributed in the cell total volume. All CD and absorption spectra have been recorded on a JASCO J-710/715 spectrometer, using 2-nm SBW, $100 \mathrm{~nm} / \mathrm{min}$ scanning speed, $0.5 \mathrm{sec}$ of integration time, and $0.2 \mathrm{~nm}$ of data pitch. Absorption spectra have been collected as HT voltage and converted into absorbance by JASCO Spectra Manager standard software. All data are blank subtracted following conventional procedures.

\section{RESULTS \\ Absorption Data}

Figure 2 (top left) shows the absorption spectra of $\Lambda$ $\mathrm{Co}(\mathrm{en})_{3} \mathrm{Cl}_{3}$ complex solution in the standard $10 \times 10 \mathrm{~mm}^{2}$ cell (solid line) compared with the one obtained with a five times more concentrated solution in the 2-mm micro cell (dotted line) (emulating in this way the fact that all absorbing molecules are occupying $20 \%$ of the cell volume). The AF effect is very evident, and the result fully justifies the name. As a further step, measurements were repeated with the less concentrated solution filled this time in the microcell (dotted line), while data related to standard cell were simply calculated by data processing (solid line). Figure 2 (bottom left) shows the overlay of the resulting absorbance spectra, now much more similar in shape, but some $\mathrm{AF}$ is still evident mainly for the 465-nm band. At this point, in order to verify the validity of our model, we have calculated the absorption spectra using eq. 1 . The $f$ factor to be used is evaluated by geometrical consideration and is 0.28 (indeed, the full beam size is 9 -mm diameter, i.e. $64 \mathrm{~mm}^{2}$, while the sample in the microcell fills a $9 \times 2 \mathrm{~mm}^{2}$ area), since the absorption of the solvent (actually the cell walls) is assumed zero, and so eq. 1 becomes

$$
T_{\operatorname{Exp}}(\lambda)=0.28 T_{\text {Sam }}(\lambda)+0.72
$$

$T_{\text {Sam }}(\lambda)$ is taken from absorption spectra in the normal $10 \times 10 \mathrm{~mm}^{2}$ cell (with multiplying factors of 5 and 1 for the two cases). Spectra calculated in this way were converted into absorbance for easy presentation. Figure 3 (left) shows the overlay of the two spectra obtained through eq. $1^{\prime}$ (solid line) together with the actual experimental ones (dotted line).

\section{Data}

Figure 2 (top right) shows the $\mathrm{CD}$ spectra taken in the same cell conditions as the absorption spectra of Figure 2 (top left). Distortions here are very evident: the major positive $\mathrm{CD}$ band is red-shifted and strongly attenuated, the negative one is virtually lost, and the positive one is weaker and red-shifted. Even the diluted sample spectrum (Fig. 2, bottom right), while much improved, still shows distortions and red shift for both the positive $\mathrm{CD}$ band. The related calculated $\mathrm{CD}$ spectra, using eq. 2 results from

$$
\mathrm{CD}_{\operatorname{Exp}}(\lambda)=0.28 \mathrm{AC}_{\mathrm{Sam}}(\lambda) / \mathrm{DC}_{\operatorname{Exp}}(\lambda)
$$

since the second term, $\mathrm{AC}_{\mathrm{Sol}}$, is zero. We do not know $\operatorname{DC}_{\operatorname{Exp}}(\lambda)$, but we know that

$$
\begin{aligned}
\mathrm{DC}_{\operatorname{Exp}}(\lambda) / \mathrm{DC}_{\mathrm{Sam}}(\lambda) & =T_{\operatorname{Exp}}(\lambda) / T_{\mathrm{Sam}}(\lambda) \quad \text { so: } \\
\operatorname{DC}_{\operatorname{Exp}}(\lambda) & =\mathrm{DC}_{\mathrm{Sam}}(\lambda)\left[T_{\operatorname{Exp}}(\lambda) / T_{\mathrm{Sam}}(\lambda)\right]
\end{aligned}
$$




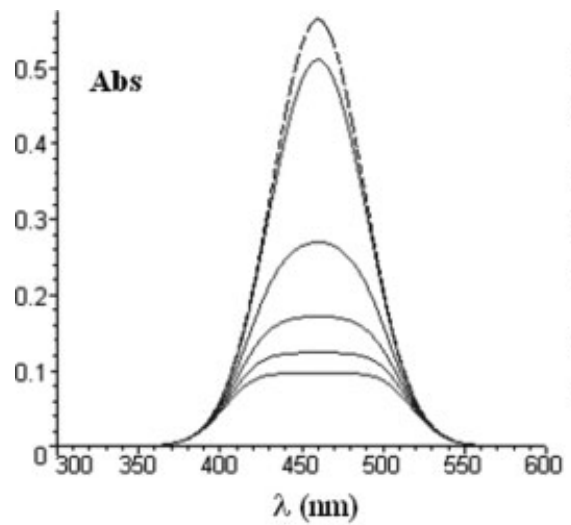

(a)

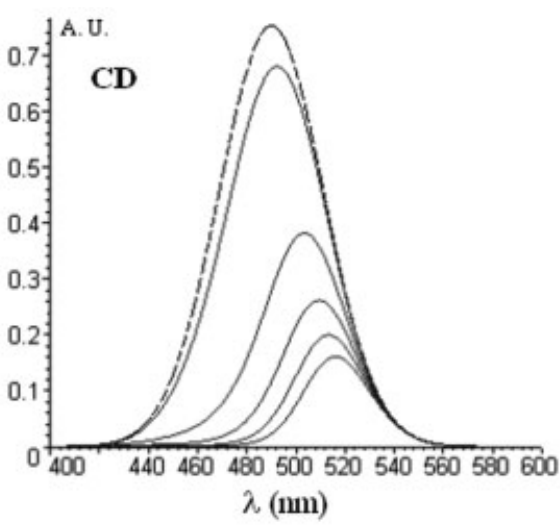

(b)

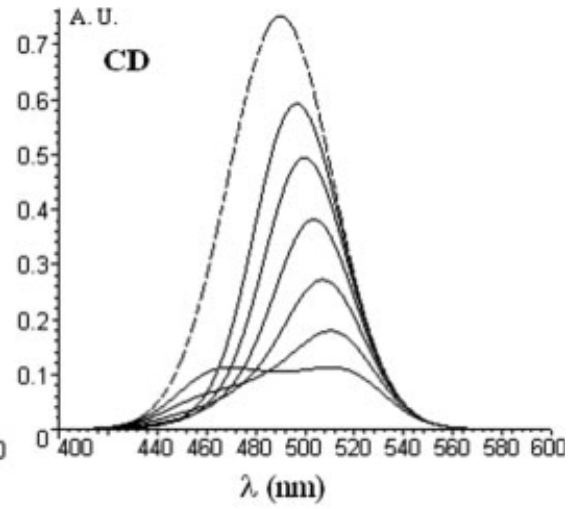

(c)

Fig. 4. (a) Inhomogeneous-sample absorptions (solid lines) and homogeneous-sample absorption (dashed line) from simulations with Gaussian bandshapes. Solid lines correspond to cells that cover only a fraction of the beam section, as in Figure $1 \mathrm{~b}$, the fraction being $f=1 / 1.1,1 / 2,1 / 3,1 / 4,1 / 5$ as peaks go down. (b) Inhomogeneous-sample CD signals (solid lines) and homogeneous-sample CD signal (dashed line) from simulation; $f$-values as in (a); still flattening increases as $f$ decreases (A.U., arbitrary units). (c) Dependence of absorption flattening on the wavelength difference of absorption and CD peaks $(f=1 / 5$, solid lines), compared with a reference homogeneous-sample CD signal (dashed line). The CD peak has been kept at 490 nm, while the absorption band has been made to vary from $490 \mathrm{~nm}$ (lower symmetric curve) to $440 \mathrm{~nm}$ (highest solid curve) by 10 -nm steps (A.U., arbitrary units).

Consequently from eq. $2^{\prime}$ and $2^{\prime \prime}$ we obtain

$$
\begin{aligned}
\mathrm{CD}_{\operatorname{Exp}}(\lambda) & =0.28\left[\operatorname{AC}_{\mathrm{Sam}}(\lambda) / \mathrm{DC}_{\mathrm{Sam}}(\lambda)\right] /\left[T_{\mathrm{Sam}}(\lambda) / T_{\operatorname{Exp}}(\lambda)\right] \\
& =0.28 \mathrm{CD}_{\mathrm{Sam}}(\lambda)\left[T_{\mathrm{Sam}}(\lambda) / T_{\operatorname{Exp}}(\lambda)\right]
\end{aligned}
$$

As mentioned earlier, $\mathrm{CD}_{\mathrm{Sam}}(\lambda)$ was calculated from the related spectra obtained in the standard cuvette by multiplying with the concentration factor. Calculated spectra on the basis of equation $\left(2^{\prime \prime \prime}\right)$ for the two concentrations are overlayed to the experimental measurements and are shown in Figure 3 (right): here the matching between experimental and theoretical data is nearly perfect. Both calculated and experimental results clearly indicate that the red wavelength shift previously observed for the main 490$\mathrm{nm}$ band (as well as spectra distortion clearly present in actual CD spectra) of this $\Lambda$-Co(en $)_{3} \mathrm{Cl}_{3}$ complex in solidstate sampling can be easily estimated:

- Considering a solution-phase sampling when $\mathrm{AF}$ is present (with our sampling model).

- By simple calculations from original $\mathrm{CD}$ and absorption spectra, applying a suitable $f$ factor.

This proves that spectra distortions observed in solid-state in many instances may come from AF (a physical reason) rather than conformational changes (chemical reason).

The AF effect can be accounted for by applying the aforementioned equations to Gaussian bandshapes. With the aid of an algebraic manipulator we have reproduced the spectra of Figure 2 (left), assuming that the absorption band centered at $460 \mathrm{~nm}$ has a Gaussian shape with $\sigma=$ $40 \mathrm{~nm}$ (Fig. 4a, dashed line). The solid lines under this curve are the prediction of the effect of confining the chiral substance to a volume $f$ times the original one $(f=1 / 1.1$, $1 / 2,1 / 3,1 / 4,1 / 5)$; accordingly, $T_{\mathrm{Sam}}$ is $T^{1 / f}$.

The analog $\mathrm{CD}$ of Figure 4a for the same values of $f$ appears in Figure 4b, where the dotted line is again the Chirality DOI 10.1002/chir
$\mathrm{CD}$ for the homogeneous sample. The solid lines are computed using eq. $2^{\prime \prime \prime}$. AF apparently causes a distortion and a shift in wavelength, because of the different positions of the absorption and $C D$ peaks. In the case presented here they are separated by $30 \mathrm{~nm}$, and the absorbance peak is at lower wavelengths. In these conditions a red shift occurs, as in Figure 2, and the shift is enhanced when $f$ tends to zero. Conversely, if the absorbance peak were at higher wavelengths with respect to the $C D$ peak, a blue shift would ensue, still growing as the curves grow less. Indeed, a shift breaks through as soon as the CD and absorbance peaks do not coincide. This is shown in Figure $4 \mathrm{c}$, where, besides the reference $\mathrm{CD}$ spectra of the homogeneous sample, a variety of curves have been produced for several wavelength peak differences (all of these latter for $f=1 / 5$ ). If the two peaks are made to coincide (at 490 $\mathrm{nm}$ in our case), then the symmetry is preserved (lowest curve). The other curves show that as the absorbance peak moves away from the $\mathrm{CD}$ one (fixed at $490 \mathrm{~nm}$, while the other peak is made to go to $440 \mathrm{~nm}$ by steps of $10 \mathrm{~nm}$ ) the $\mathrm{AF}$ effect, no longer concealed by symmetry, becomes smaller and smaller. At about $400 \mathrm{~nm}$, the curve (not represented) would hardly be distinguishable from the dashed line.

Alongside with the earlier derivation, we would like to make the following comments:

i. AF-induced distortions in $\mathrm{CD}$ spectra are more severe than in the absorption ones; in particular, $\mathrm{CD}$ bands are not observed at the same wavelength as absorption ones, since $\mathrm{AF}$ induces a wavelength shift of the CD bands;

ii. In simple cases, it is possible to provide a mathematical model like the one we have provided, to account for the distortion effects, starting from the $\mathrm{CD}_{\mathrm{Sam}}(\lambda)$ and the absorption spectra $T_{\mathrm{Sam}}(\lambda)$ in solution. When the $f$ factor is difficult to estimate, a trial with different 

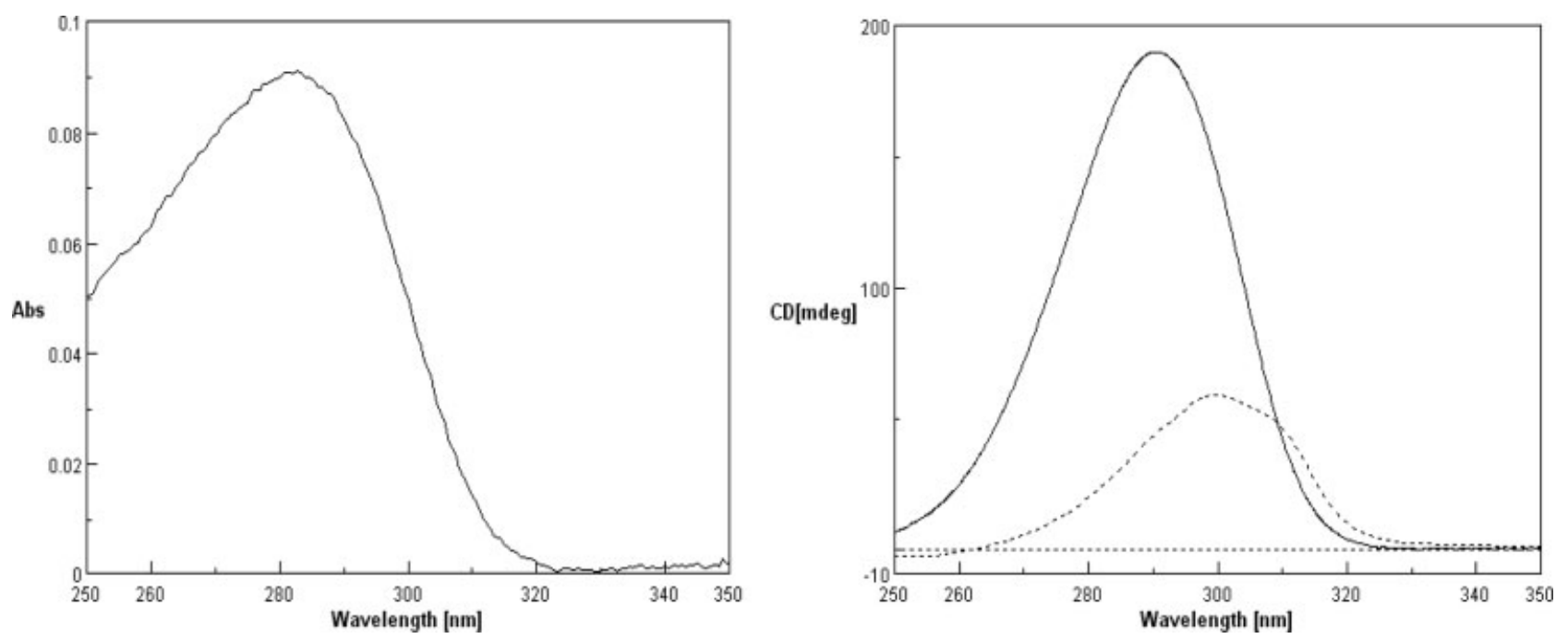

Fig. 5. Left: absorption spectra of the ammonium $d-10$ camphorsulphonate (ACS) aqueous solution. Right: CD spectra of aqueous solution of ACS (一) compared to $\mathrm{KCl}$ pellet (-- -).

$f$ values will give proper indications. But remember to correct also absorption and CD spectra in solution for a concentration effect equal to $1 / f$;

iii. In any case, the simplest and safest way to eliminate or at least reduce AF effects is sample dilution, if sensitivity allows;

iv. The importance to collect absorption spectra together with CD spectra must be stressed, and obviously absorption data collection should be made on the same instrument with identical sampling beam geometry as CD.

\section{CONCLUSIONS}

Herewith we have been showing that $\mathrm{AF}$ is the reason for wavelength shifts and distortions in the $\mathrm{CD}$ spectra of $\Lambda$-Co(en $)_{3} \mathrm{Cl}_{3}$ complex inhomogeneous solutions with respect to the $\mathrm{CD}$ spectra of clean solutions. We opine that the same reason may explain similar $\mathrm{CD}$ spectra distortions reported for other samples when comparing solution to solid-state data. For example, Watanabe et al. in a poster paper $^{11}$ at the CD-2005 Conference showed that in the well-known ammonium $d-10$ camphorsulphonate spectra taken in $\mathrm{KCl}$ pellet, the $290-\mathrm{nm}$ band is somehow redshifted with respect to what was observed in water solution (Fig. 5); also in this case, the absorption spectra in solution shows that the absorption band is at lower wavelengths than the $\mathrm{CD}$ one: this will explain the red shift of the $\mathrm{CD}$ solid-state spectra.

The proposed method to estimate $\mathrm{AF}$ with a microcell can be used in several cases. However, when spectra need to be run in the shorter wavelength UV range with short pathlength cuvettes, this approach is not possible (since no commercial short-path microcell is available), and so a partial filling of the cuvette may be used as an alternative (Fig. 1 , right). However, in these cases, we have no way to take into account solvent/buffer absorption which is likely to be sizeable in the low wavelength UV range. A further special solid-sampling case would be film analysis. In this case, additional cautions are necessary, since macroscopic anisotropies $^{12}$ may play an additional role in spectra distortion.

May we extend the earlier approach to solution-phase spectra of suspensions, membranes, and aggregates? We think we may, since they are a sort of solid-state spectroscopy in which the solid sample is dispersed in a liquid. AF will play a major role with these samples, but also light scattering (LS) will give contribution. If particles are achiral, LS will only reduce the number of photons collected by the photomultiplier tube and impair the signal-to-noise ratio, but with little influence on $\mathrm{CD}$ spectral shape. ${ }^{13}$ But if particles are chiral, differential scattering for the right and left circularly polarized components will take place.

So far the only practical way to improve CD data has been to place the detector as close as possible to the sample, to increase the solid angle ${ }^{14}$ (i.e. collecting as far as possible the forward scattering component). Several models to compensate $\mathrm{AF}$ in these cases were reported, mainly based on the regular absorption (or the photomultiplier tube high voltage, which is directly related to absorbance), as in a brilliant case recently published. ${ }^{15}$ However, as we have shown earlier, the problem comes up when the CD band is not at the same wavelength as the absorption one; in these cases, the $\mathrm{CD}$ spectral shape correction may be arbitrary. A series of measurements at different dilutions seems probably the most reliable way to reduce $\mathrm{AF}$ artifacts, or at least to estimate the influence of $\mathrm{AF}$ in spectral data.

Finally we would like to make the following remark: even very common $\mathrm{CD}$ thermal melting experiments of biomolecules may be influenced by $\mathrm{AF}$, particularly when dealing with short pathlength cells, where it is common to see air bubbles growing within the sample when the temperature is increasing. These phenomena will give, almost by definition, AF effects, prone to alter spectral shape and/or to limit data fidelity at monitoring wavelength. The same sort of problems may be present dealing with frozen samples. 


\section{LITERATURE CITED}

1. Duysens LMN. The flattening of the absorption spectrum of suspensions, as compared to that of solutions. Biochim Biophys Acta 1956;19:1-12.

2. Wittung P, Kajanus J, Kubista M, Malmstrom BG. Absorption flattening in the optical spectra of liposome-entrapped substances. FEBS Lett 1994;352:37-40.

3. Gordon DJ, Holzwarth G. Optical activity of membrane suspensions: Calculation of artfacts by Mie scattering theory. Proc Natl Acad Sci USA 1971;68:2365-2369.

4. Bustamante C, Maestre MF. Statistical effects in the absorption and optical activity of particulate suspensions. Proc Natl Acad Sci USA 1988;85:8482-8486.

5. Grosjean M, Legrand M. Appareils de mesure du dichroisme circulaire dans le visible et l'ultraviolet, Comptes rendus, 251, 1960: 21502152. Dichrograph Philips Sci Ind Bull 1965;4:1-8.

6. Broomhead JA, Dwyer FP, Hogarth JW. Resolution of the tris(ethylendiamine) cobalt(III) ion. In: Rochow EG, editor. Inorganic synthesis, Vol. 6. New York: McGraw Hill; 1960. p 183-188.

7. JASCO, Application Notes $\mathrm{N}^{\circ} 1$ 1971;2-4.

8. Kuroda R. Solid-state CD: application to inorganic and organic chemistry. In: Berova N, Nakanishi K, Woody RW, editors. Circular dichroism: Principles and applications. New York: Wiley; 2000. p 159-184.
9. Castiglioni E, Albertini P. An integrating sphere to measure $\mathrm{CD}$ from difficult samples. Chirality 2000;12:291-294.

10. Billotti I, Biscarini P, Castiglioni E, Ferranti F, Kuroda R. Reflectance circular dichroism of solid state chiral coordination compounds. Chirality 2002;14:750-756.

11. Watanabe M, Hayakawa H, Takakuwa T, Masago H, Wada Q. Expansion of pellet method for the CD measurement to V-UV region. In 10th International Conference on CD, August 2005, Destin, Florida, USA. Poster 47.

12. Harada T, Kuroda R. Circular dichroism measurement of a protein in dried thin films. Chem Lett 2002:326-327.

13. Wallace BA, Mao D. Circular dichroism analyses of membrane proteins: an examination of light scattering and absorption flattening in large membrane vescicles and membrane sheets. Anal Biochem 1984;142:317-328.

14. Schneider AS, Harmatz D. An experimental method correcting for absorption flattening and scattering in suspensions of absorbing particles: circular dichroism and absorption spectra of hemoglobin in situ in red blood cells. Biochemistry 1976;15:4158-4162.

15. Ganesan A, Price Nc, Kelly SM, Petry I, Moore BD, Halling PJ. Circular dichroism studies of subtilisin Carlsberg immobilised on micron sized silica particles. Biochem Biophys Acta 2006;1764:1119_ 1125 . 